\title{
Article \\ Establishing a New ECMO Referral Center Using an ICU-Based Approach: A Feasibility and Safety Study
}

\author{
Ryszard Gawda ${ }^{1, * \mathbb{D}}$, Maciej Piwoda ${ }^{1}$, Maciej Marszalski ${ }^{1}$, Katarzyna Lyp ${ }^{2}$, Jolanta Piwoda ${ }^{3}$, Magdalena Maj ${ }^{3}$, \\ Maciej Gawor ${ }^{3}$, Maciej Molsa ${ }^{1}$, Marek Pietka ${ }^{3}$ and Tomasz Czarnik ${ }^{1}$ (D)
}

1 Department of Anesthesiology, Intensive Care and Regional ECMO Center, Institute of Medical Sciences, University of Opole, 45-401 Opole, Poland; mpiwoda@mp.pl (M.P.); maciek.marszalski@gmail.com (M.M.); molsamaciej@gmail.com (M.M.); tczarnik@mac.com (T.C.)

2 Department of Family Medicine and Public Health, Faculty of Medicine, University of Opole, 45-052 Opole, Poland; katarzyna.lyp@uni.opole.pl

3 Department of Anesthesiology, Intensive Care and Regional ECMO Center, University Hospital in Opole, 45-401 Opole, Poland; jo_linka@interia.pl (J.P.); truchan.m@gmail.com (M.M.); macgawor@interia.pl (M.G.); mpietka@gmail.com (M.P.)

* Correspondence: rgawda@wp.eu

\section{check for}

updates

Citation: Gawda, R.; Piwoda, M.;

Marszalski, M.; Lyp, K.; Piwoda, J.;

Maj, M.; Gawor, M.; Molsa, M.;

Pietka, M.; Czarnik, T. Establishing a New ECMO Referral Center Using an ICU-Based Approach: A Feasibility and Safety Study. Healthcare 2022, 10, 414. https://doi.org/10.3390/

healthcare10030414

Academic Editors: Luigi Vetrugno and Cristian Deana

Received: 18 December 2021

Accepted: 21 February 2022

Published: 22 February 2022

Publisher's Note: MDPI stays neutral with regard to jurisdictional claims in published maps and institutional affiliations.

Copyright: (c) 2022 by the authors. Licensee MDPI, Basel, Switzerland. This article is an open access article distributed under the terms and conditions of the Creative Commons Attribution (CC BY) license (https:// creativecommons.org/licenses/by/ $4.0 /)$.

\begin{abstract}
Background: A high-volume center with a multidisciplinary team is regarded as the optimal place for providing extracorporeal membrane oxygenation (ECMO). We hypothesize that an ECMO center can also be successfully created and subsequently developed entirely by intensivists in a mid-size mixed intensive care unit (ICU). Methods: A model was created for setting up a new ECMO referral center within the structure of an existing mixed ICU in a tertiary hospital. A retrospective analysis was carried out of the first 33 patients treated in the initial period of the center's activity, from mid 2018 to the end of 2020. Results: An ECMO center was established and developed entirely based on the resources of an existing mixed ICU. Thirty-three patients were treated. They had an overall survival rate at 90 days of $60.6 \%$. In veno-venous (VV) mode ECMO duration, ICU length of stay, and SOFA score were significantly higher than in veno-arterial mode. No significant differences in clinical characteristics were observed between survivors and non-survivors on VV-ECMO. Conclusions: A regional ECMO center can be set up as an integral part of a mixed ICU in a tertiary hospital. Extracorporeal therapy, such as continuous renal replacement therapy and mechanical ventilation can be managed entirely by intensivists. Further studies are needed to show that the ICU-based approach to setting up a new ECMO center is no less effective than the multidisciplinary approach.
\end{abstract}

Keywords: critical care; healthcare; intensive care unit; extracorporeal membrane oxygenation; ECMO

\section{Introduction}

Extracorporeal membrane oxygenation (ECMO) is one of the most advanced techniques used in acute respiratory, as well as circulatory, failure [1]. In 2020, $492 \mathrm{ECMO}$ centers were registered at the Extracorporeal Life Support Organization (ELSO), the largest worldwide organization consociating health care workers involved in extracorporeal therapy [2]. Experts argue that the optimal conditions for this kind of treatment are best provided in high-volume dedicated centers, since the outcomes are linked with the volume of patients $[3,4]$. In reality, the majority of ECMO procedures are performed in low-volume centers $[5,6]$.

There are numerous requirements to fulfil to successfully set up a new ECMO center. Obtaining an institutional commitment and financial support seems to be one of the most significant. How to form an ECMO team and where to locate a new ECMO center in a hospital area is another fundamental problem. Additionally, recommendations suggest creating a multidisciplinary group consisting of various specialists, because of the complicated nature of extracorporeal therapy [7-10]. 
Reports on practical methods for setting up new ECMO centers are limited [11,12]. To date, there have been no reports describing the establishment of ECMO centers solely from the perspective of an individual mixed intensive care unit (ICU). We hypothesize that an ECMO center guided only by intensivists has the potential to be a full value ECMO referral center. Our primary aim was, thus, to describe the main steps required for the establishment of a new ECMO center and to present a concept of an ICU-based approach to conducting ECMO therapy. Our secondary aim was to outline the patient demographic data and outcomes achieved in the initial period of the center's activity.

\section{Materials and Methods}

This is a feasibility and safety study on the setting up of a new ECMO referral center in a tertiary hospital. The study design was approved by the Regional Ethics Committee (date of approval: 11 June 2021, decision number: 335/2021). The requirement for patient written informed consent was waived.

Our ECMO center was established solely as a part of the existing mixed 11-bed ICU of the University Hospital in Opole, Poland. This is a level 3 ICU with intensivists constantly present, the nurse to patient ratio is 1:2, and the full spectrum of organ dysfunction is treated. Besides the mixed ICU, critically ill patients in our hospital are also treated in the cardiosurgical ICU and pediatric/neonatal ICU. These ICUs are administratively separate structures. Staff at our ICU consist only of intensivists, registered nurses, and physiotherapists. The ICU serves approximately 450 patients a year.

When our ECMO center was set up, there was no coordinated ECMO system in Poland. Moreover, ECMO therapy was practically inaccessible in Opole Voivodeship. No specific financial support for the ECMO program was initially allocated, except for funds to purchase the first ECMO machine. The institutional commitment was mainly limited to the approval of administrative routines. All ECMO procedures are financed intrinsically by the Polish National Health Fund.

Chronologically, the overall development of the ECMO center was divided into four main steps: initialization, training, preparation, and activation. A timeline of the center's progress is shown in Figure 1.

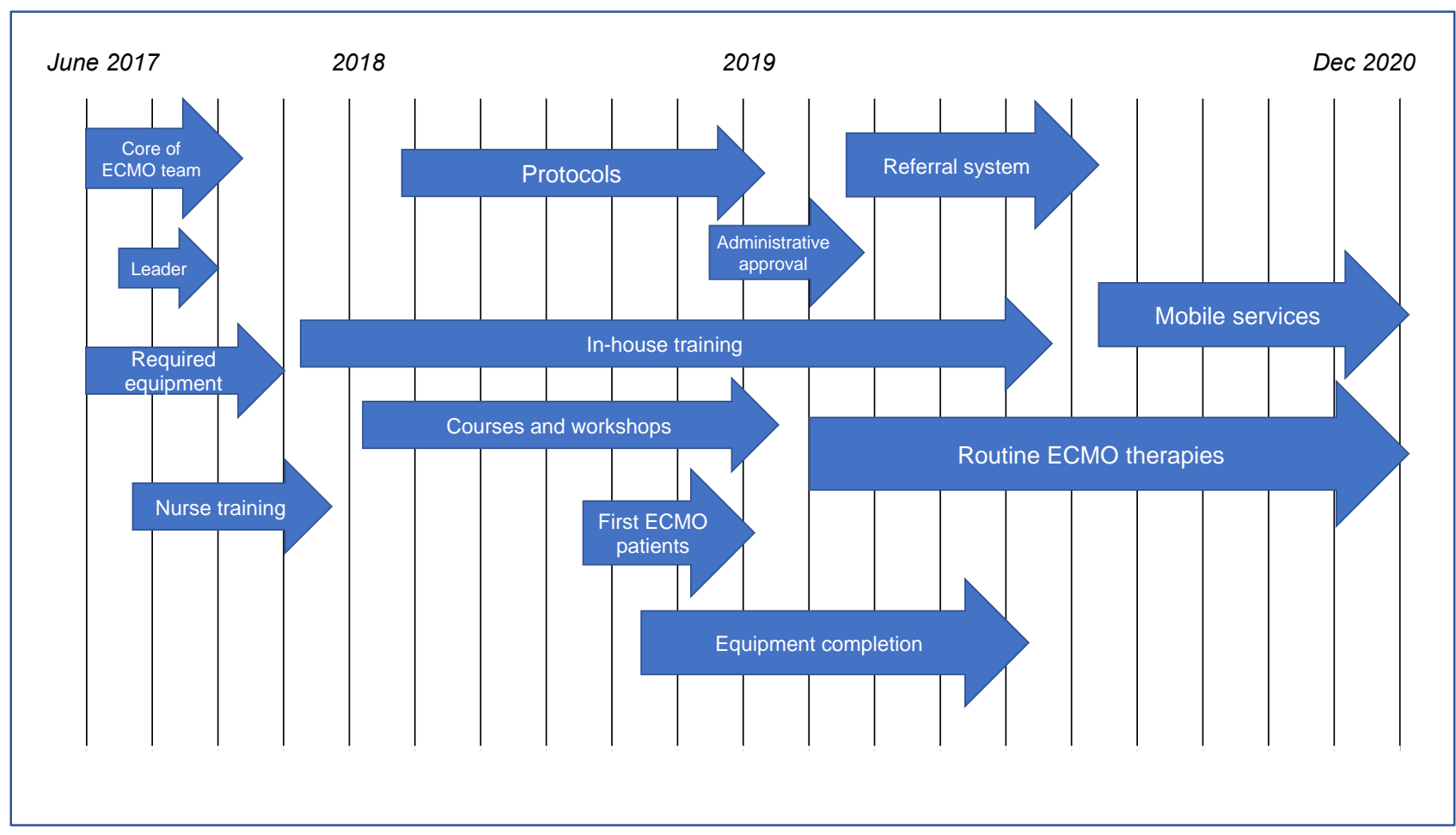

Figure 1. Timeline of the ECMO center progression. 


\subsection{Initialization}

The concept of establishing an ECMO program was raised at the beginning of 2017 by two intensivists (T.C. and R.G.) before scheduled updating of the ICU equipment. After institutional approval, a sum of EUR 90,000 was allocated for the purchase of an ECMO machine (Cardiohelp, Maquet, Germany) and to finance intensivists attending simulation workshops in November 2017 (Rastatt, Germany). A group of six board-certified intensivists of the existing ICU formed the ECMO team, and a leader (T.C.) was designated.

\subsection{Training}

In the first half of 2018, the ECMO team attended numerous internal training sessions, including (a) thorough theoretical preparation, (b) ECMO machine management, including basic servicing and priming a circuit, and (c) simulation of typical critical scenarios. The registered nurses of our ICU attended ECMO courses held exclusively for ECMO nurses and were subsequently trained by the ECMO team physicians. During 2018, team members attended courses and workshops in external centers, including the ELSO ECMO course at Karolinska University Hospital (Stockholm, Sweden), Paris Rescue Team Course (Paris, France), and ECMO Simulation Workshops (Poznan, Poland).

\subsection{Preparation}

In the second half of 2018, the ECMO team announced that they were ready to perform ECMO therapy on some selected patients hospitalized in the University Hospital. Two veno-venous ECMO (VV-ECMO) and two veno-arterial ECMO (VA-ECMO) therapies were conducted to the end of 2018. Internal training was continued in 2018 and a second ECMO machine (Cardiohelp, Maquet, Germany) was bought with financial support from the local government.

\subsection{Activation}

Technically, the local government and hospital authorities inaugurated the Regional ECMO Center in Opole in January 2019. A short local media campaign was conducted, and the district hospitals were informed of the center's activation. The center served a population of the Opole area (one million citizens). At the end of 2019, the center extended its activity to patients from adjacent regions. Mobile services, including both ground transportation (at the beginning of 2020) and a helicopter (at the end of 2020), were thus introduced. Finally, a third ECMO machine was bought.

Establishing and developing an ECMO center was complex. The most significant milestones of the ECMO center activity are shown in Figure 2.

\subsection{Protocols}

To qualify for VV-ECMO, we incorporated criteria used in the EOLIA trial as core indications [13]. However, the final patient qualification is individualized and based on the clinical context (actual clinical data of the patient or on existing therapy trends). Several internal protocols were incorporated into daily practice, including plans of mechanical ventilation, patient's weaning, anticoagulation proceedings, and pharmacotherapy adjustment. Protocols for critical situations (oxygenator dysfunction, extracorporeal system failure, accidental decannulation, recirculation) during ECMO were prepared and practiced. Protocols for intra- and inter-hospital ECMO transportations were also prepared.

\subsection{Patient Treatment}

VA-ECMO is accessible only for in-hospital patients. Cannulation is performed percutaneously both by ECMO intensivists in the ICU and collectively with interventional cardiologists in the catheterization laboratory using real-time ultrasound (US) guidance. In the case of indications of left ventricle unloading, an Impella device or intra-aortic balloon pump are inserted percutaneously by an interventional cardiologist. An arterial cannula is only removed in the operating theatre by a vascular surgeon. 


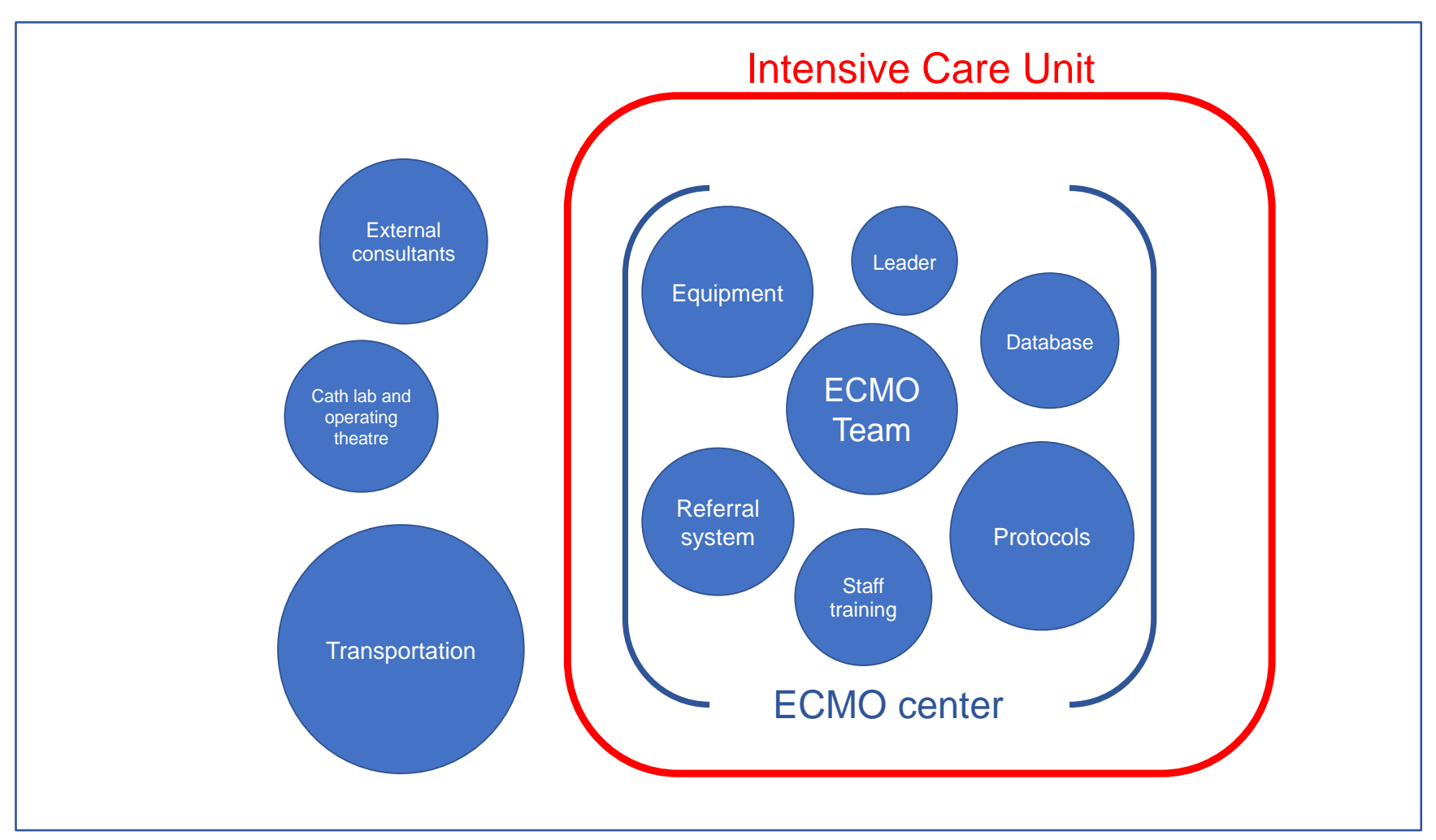

Figure 2. Components of the ICU-based ECMO center.

VV-EMCO is provided both for in- and out-of-hospital patients. Before cannulation, an ECMO intensivist performs a routine transthoracic echocardiogram (TTE) and ultrasound examination of both the lungs and great vessels. Cannulation is performed solely percutaneously using real-time US-guidance, and the position of the pericardiac cannula is confirmed by an ECMO intensivist with the use of TTE or a transesophageal echocardiogram (TEE).

Both methods of extracorporeal therapy are conducted in the ICU. Only intensivists (ECMO team members) are responsible for all aspects of the extracorporeal therapy, e.g., priming the circuit, patient monitoring and examination, ECMO machine handling, treatment assessment, weaning, and procedure termination. Anticoagulation is provided with continuous administration of unfractionated heparin. Protective mechanical ventilation is applied to maintain a tidal volume below $6 \mathrm{~mL} / \mathrm{kg}$ of predicted body weight with plateau pressure below $25 \mathrm{~cm} \mathrm{H}_{2} \mathrm{O}$, and positive end-expiratory pressure around $10 \mathrm{~cm}$ $\mathrm{H}_{2} \mathrm{O}$. All routine procedures of up-to-date intensive care are provided, including daily rounds, patient-tailored feeding, sedation holiday, and patient early mobilization. After decannulation, the patients are treated accordingly, still in the same ICU, by ECMO team members, until the patients can be safely transferred out of the intensive care environment.

\subsection{Staff and Equipment}

All extracorporeal therapies are managed solely by intensivists who are members of the ECMO team. While, 10 intensivists have been trained and are fully involved in the ECMO center activity. The ECMO team is supported by registered nurses working at the bedside in the ICU. As in other critically ill patients, physiotherapists are involved in the patient's early mobilization. No additional health professionals (perfusionists, surgeons, cardiologists, respiratory therapists, nutritionists, pharmacists, etc.) are involved in the extracorporeal therapy. A vascular surgeon, interventional cardiologist, and cardiac surgeon can be called upon at an hour's notice if required. 


\subsection{Referral System}

A referral system for patients treated outside the University Hospital in Opole was created. This is based both on a website and two dedicated telephone lines. The data on the reported patients are analyzed collectively by at least two ECMO team physicians and the final decision is taken within $30 \mathrm{~min}$. Four resolutions are then applied: (1) qualification with ground or rotor-wing transportation of the patient executed by an emergency medical service (EMS), (2) qualification and activation of the mobile ECMO team, activating the ECMO procedure at the referring hospital with subsequent transportation on ECMO either by ground or air, (3) temporal disqualification with recommendations regarding optimization of therapy and repeated consultation the next day, (4) ultimate disqualification. The COVID-19 pandemic imposed a specific approach for the qualification of SARS-CoV-2 infected patients, in line with specific ELSO guidelines [14].

\subsection{ECMO Transportation}

For transportation, strict cooperation with the EMS was established. The mobile ECMO team consisted of two ECMO team intensivists (alternatively an ECMO intensivist with residency in intensive care medicine) and two paramedics from the EMS. An ambulance or helicopter is provided by EMS. To date, the mobile ECMO team has serviced patients only on VV-ECMO. Currently, there is no coordinated cardiogenic shock referral system in Opole Voivodeship. The ECMO team is equipped with everything necessary for ECMO commencement including an ECMO machine, cannulas, respirator, and an ultrasound machine with TEE probe. After arrival at the referring ICU, the ECMO team performs cannulation and transfers the patient started on extracorporeal therapy to the Regional ECMO Center in Opole. Members of the ECMO team are insured against unforeseen accidents. During the COVID-19 pandemic, our mobile ECMO team performed ground and rotor-wing aircraft transportation of SARS-CoV-2 infected patients on VV-ECMO.

\subsection{Database}

All patients are entered anonymously into two separate databases created as Excel spreadsheets. The first database comprises patients started on VA-ECMO therapy. The second database comprises patients treated with VV-ECMO. Patient baseline data are collected on therapy, complications, and outcomes. A phone-based follow-up of every patient is conducted to assess the outcomes at 28 and 90 days after decannulation. Every patient is also recorded on the ELSO registry.

\subsection{Statistical Methods}

Data analysis was performed using Statistica 13.3 PL (TIBCO Software Inc., Palo Alto, CA, USA). For continuous variables, the median and IQR $\left(Q_{1}-Q_{3}\right)$ were calculated, while the discrete variables were expressed as percentages, and cardinality $(n)$ was taken into account and verified with the $x^{2}$ test. The normality of the distribution was verified using the Shapiro-Wilk test. In the case of non-compliance of the distribution of variables with the normal distribution, the Mann-Whitney U test (with corrections for continuity) was used, and in the remaining cases, the T test was applied. Statistical differences were considered significant at $p<0.05$.

\section{Results}

The development of all stages of the Regional ECMO Center in Opole have been completed within 28 months. As a result, VV-ECMO therapy is now provided for the population of approximately 3 million. VA-ECMO therapy is ensured only for the patients of the University Hospital. Both methods of extracorporeal therapy are performed entirely within the existing mixed ICU and are conducted only by intensivists. This way, ECMO is considered as another modality in the ICU, alongside mechanical ventilation and renal replacement therapy. Availability of on-call cardiovascular specialists and participation 
of EMS in ECMO transportation are the only constituents of our ECMO center that are derived from the outside of the ICU.

From July 2018 to December 2020, 33 patients were treated: 22 on VV-ECMO (including eight cases of COVID-19) and 11 on VA-ECMO. Baseline characteristics of all patients are presented in Table 1. Median support time on VV-ECMO was $365 \mathrm{~h}$, which was significantly higher than the median time of $49 \mathrm{~h}$ on VA-ECMO. Significant differences were also observed between the VV-ECMO and VA-ECMO groups regarding SOFA score and ICU length of stay. Overall survival rate at ICU discharge was $63.6 \%$, survival rate at 28 and at 90 days after decannulation was $63.6 \%$ and $60.6 \%$, respectively.

Table 1. Baseline patient characteristics.

\begin{tabular}{ccccc}
\hline Variable & All Patients $(\boldsymbol{n}=\mathbf{3 3})$ & $\begin{array}{c}\text { VV-ECMO } \\
(\boldsymbol{n}=\mathbf{2 2})\end{array}$ & $\begin{array}{c}\text { VA-ECMO } \\
(\boldsymbol{n}=\mathbf{1 1})\end{array}$ & $\boldsymbol{p}$ Value \\
\hline Age, years & $52(40-59)$ & $52(39-58)$ & $53(44-68)$ & 0.2381 \\
Female sex, $n(\%)$ & $8(24.2)$ & $4(18.2)$ & $4(36.4)$ & 0.4727 \\
Weight, kg & $80(70-99)$ & $85(70-99)$ & $72(68-89)$ & 0.1516 \\
BMI, kg/cm & $26(23-31)$ & $26(23-33)$ & $25(24-28)$ & 0.1799 \\
SOFA score & $9(7-12), n=30$ & $7(6-12)$ & $11(10-14), n=8$ & 0.0051 \\
CRRT, $n(\%)$ & $16(48.5)$ & $11(50)$ & $5(45.5)$ & 0.9020 \\
ECMO duration, hours & $277(56-464)$ & $365(218-496)$ & $49(12-113)$ & 0.0006 \\
ICU length of stay, days & $17(6-33)$ & $19(9-37)$ & $6(2-20)$ & 0.0355 \\
\hline
\end{tabular}

Note: data are presented as median (IQR), $n(\%), n$ (where data are not available for all patients). Abbreviations: BMI—body mass index, SOFA—Sequential Organ Failure Assessment, CRRT—continuous renal replacement therapy, ECMO_-extracorporeal membrane oxygenation, ICU-intensive care unit.

Characteristics of the VV-ECMO therapy are shown in Table 2. No significant differences were observed between survivors and non-survivors. A main indication for ECMO was acute respiratory distress syndrome (ARDS) caused by viral (COVID-19, 8 patients, influenza, 2 patients) or bacterial infection (8 patients). In four cases of ARDS, pneumonia was of unknown etiology. The complications during VV-ECMO were as follows: nasopharyngeal bleeding ( 4 patients), pneumothorax ( 2 patients), and bleeding from cannulation sites (one patient). Four patients were cannulated in the external hospitals by the mobile ECMO team and subsequently transported to the center in 2020. No disturbances in extracorporeal therapy were observed during transportation.

Table 2. Characteristics of patients on VV-ECMO.

\begin{tabular}{|c|c|c|c|c|}
\hline & \multirow{2}{*}{ All Patients $(n=22)$} & \multicolumn{2}{|c|}{ Status at 90 Days after ECMO } & \multirow{2}{*}{$p$ Value } \\
\hline & & Alive $(n=13)$ & Dead $(n=9)$ & \\
\hline \multicolumn{5}{|l|}{ Mode of circuit } \\
\hline Jugulo-femoral, $n(\%)$ & $17(77.3)$ & $10(76.9)$ & $7(77.8)$ & 0.6381 \\
\hline Femoro-femoral, $n(\%)$ & $5(22.7)$ & $3(23.1)$ & $2(22.2)$ & 0.6381 \\
\hline Admission cannula, Fr & $25(23-25) ; n=15$ & $25(23-25) ; n=8$ & $25(24-25) ; n=7$ & 0.6754 \\
\hline Return cannula, Fr & $23(22-24) ; n=15$ & $23(22-25) ; n=8$ & $23(21-23) ; n=7$ & 0.2238 \\
\hline \multicolumn{5}{|l|}{ Indications for ECMO } \\
\hline ARDS of viral etiology, $n(\%)$ & $10(45.5)$ & $6(46.1)$ & $4(44.4)$ & 0.4789 \\
\hline ARDS of bacterial etiology, $n(\%)$ & $8(36.4)$ & $5(38.4)$ & $3(33.3)$ & 0.4423 \\
\hline ARDS of unknown etiology, $n(\%)$ & $4(18.2)$ & $2(15.4)$ & $2(22.2)$ & 0.4309 \\
\hline \multicolumn{5}{|l|}{ Ventilation before ECMO } \\
\hline Peak pressure, $\mathrm{cm} \mathrm{H}_{2} \mathrm{O}$ & $31(29-34) ; n=20$ & $33(30-35) ; n=12$ & $30(28-32) ; n=8$ & 0.3788 \\
\hline Plateau pressure, $\mathrm{cm} \mathrm{H}_{2} \mathrm{O}$ & $29(26-32) ; n=16$ & $30(27-34) ; n=9$ & $26(26-30) ; n=7$ & 0.3350 \\
\hline Driving pressure, $\mathrm{cm} \mathrm{H}_{2} \mathrm{O}$ & $16(13-18) ; n=19$ & $16(14-19) ; n=11$ & $15(12-17) ; n=8$ & 0.4101 \\
\hline $\mathrm{PEEP}, \mathrm{cm} \mathrm{H} \mathrm{H}_{2} \mathrm{O}$ & $12(12-14)$ & $14(12-15)$ & $12(11-14)$ & 0.1794 \\
\hline $\mathrm{FiO}_{2}$ & $0.8(0.7-1)$ & $0.8(0.6-1)$ & $0.8(0.8-1)$ & 0.3294 \\
\hline Tidal volume, mL/kg PBW & $5(4-5) ; n=13$ & $5.7(4-6) ; n=7$ & $4.9(4-5) ; n=6$ & 0.2644 \\
\hline Prone position, $n(\%)$ & $8(36.4)$ & $6(46.1)$ & $2(22.2)$ & 0.4861 \\
\hline
\end{tabular}


Table 2. Cont.

\begin{tabular}{|c|c|c|c|c|}
\hline & \multirow{2}{*}{ All Patients $(n=22)$} & \multicolumn{2}{|c|}{ Status at 90 Days after ECMO } & \multirow{2}{*}{$p$ Value } \\
\hline & & Alive $(n=13)$ & Dead $(n=9)$ & \\
\hline \multicolumn{5}{|l|}{ Intervention on ECMO } \\
\hline Mechanical ventilation, hours & $365(179-496)$ & $336(168-500)$ & $432(215-476)$ & 0.7528 \\
\hline Sedation, hours & $306(132-470) ; n=19$ & $306(132-458) ; n=11$ & $336(170-467) ; n=8$ & 0.9099 \\
\hline Neuromuscular blockade, $n(\%)$ & $14(63.6)$ & $9(69.2)$ & $5(55.5)$ & 0.8377 \\
\hline Tracheostomy, $n(\%)$ & $13(59.1)$ & $8(61.5)$ & $5(55.5)$ & 0.8726 \\
\hline
\end{tabular}

Note: data are presented as median (IQR), $n(\%), n$ (where data are not available for all patients). Abbreviations: Fr-diameter in French catheter scale, ARDS-acute respiratory distress syndrome, PEEP—positive endexpiratory pressure, $\mathrm{FiO}_{2}$ - fraction of inspired oxygen, $\mathrm{PBW}$ - predicted body weight, ECMO — extracorporeal membrane oxygenation.

The parameters of the VA-ECMO therapy are presented in Table 3. The indications for VA-ECMO were as follows: myocardial infarction (4 patients), myocarditis (2 patients), intra-hospital cardiac arrest (2 patients), and high-risk percutaneous coronary intervention (3 patients). Six patients were treated with a high dose of noradrenaline before ECMO placement. Complications during VA-ECMO included left ventricle distention (4 patients), bleeding from cannulation sites (4 patients), and lower limb ischemia (2 patients).

Table 3. Patient characteristics on VA-ECMO.

\begin{tabular}{|c|c|}
\hline & VA-ECMO $(n=11)$ \\
\hline \multicolumn{2}{|l|}{ Mode of circuit } \\
\hline Femoro-femoral, $n$ (\%) & $8(72.7)$ \\
\hline Jugulo-femoral, $n(\%)$ & $3(27.3)$ \\
\hline Admission cannula, Fr & $25(25-27) ; n=6$ \\
\hline Return cannula, Fr & $18(17-19) ; n=6$ \\
\hline \multicolumn{2}{|l|}{ Indications for ECMO } \\
\hline Myocardial infarction, $n(\%)$ & $4(36.3)$ \\
\hline Myocarditis, $n(\%)$ & $2(18.2)$ \\
\hline Cardiac arrest, $n(\%)$ & $2(18.2)$ \\
\hline High-risk coronary angioplasty, $n(\%)$ & $3(27.3)$ \\
\hline \multicolumn{2}{|l|}{ Parameters before ECMO } \\
\hline $\mathrm{MAP}, \mathrm{mm} \mathrm{Hg}$ & $50(35-56) ; n=8$ \\
\hline $\mathrm{HR}$ & $96(86-115) ; n=6$ \\
\hline EF LV, percentage & $15(5-20) ; n=9$ \\
\hline Creatinine, $\mathrm{mg} / \mathrm{dL}$ & $1.25(0.8-1.6) ; n=10$ \\
\hline Arterial, $\mathrm{pH}$ & $7.1(7.1-7.2) ; n=6$ \\
\hline Lactate, $\mathrm{mmol} / \mathrm{L}$ & $10(7-13) ; n=5$ \\
\hline \multicolumn{2}{|l|}{ Medication before ECMO } \\
\hline Norepinephrine, $\mu \mathrm{g} / \mathrm{kg} / \mathrm{min}$ & $0.2(0-0.3) ; n=10$ \\
\hline Dobutamine, $\mathrm{mg} / \mathrm{kg} / \mathrm{min}$ & $0(0-0) ; n=10$ \\
\hline Adrenaline, $\mu \mathrm{g} / \mathrm{kg} / \mathrm{min}$ & $0(0-0) ; n=10$ \\
\hline \multicolumn{2}{|l|}{ Mode of LV venting } \\
\hline IABP, $n(\%)$ & $1(9.1)$ \\
\hline Atrial septostomy, $n(\%)$ & $1(9.1)$ \\
\hline Impella, $n(\%)$ & $2(18.2)$ \\
\hline
\end{tabular}

\section{Discussion}

Our ECMO center was created over two years based solely on an existing mixed ICU in a 500 bed University Hospital in Opole. It took two and a half years for the center to operate routinely. A similar amount of time has been reported by other centers developed in tertiary hospitals [15]. The survival rates of our patients are comparable to those reported by some high-volume centers $[2,12,16]$. 
ECMO centers may be set up as a result of national healthcare strategies that involve creating network centers [17]. Having the support of local authorities is key for new ECMO center creation, particularly in its early stages [11]. Setting up our ECMO center was a totally bottom-up initiative, with financial support limited only to purchasing the ECMO machines required.

There is no consensus as to where a new ECMO center should be located [9]. Experts indicate that immediate access to various specialists should be guaranteed; thus, a tertiary hospital is considered as an optimal option [15]. In hospitals, cardiothoracic departments are often used as a location for a new ECMO center. This is above all due to the fact that extracorporeal circulation is performed routinely during open heart surgery [18]. Various types of ICUs, mainly cardiothoracic and cardiac, may be the location for a new ECMO center. A primary driving force in setting up our ECMO center was a group of intensivists who strongly felt that there would be considerable benefits. A natural consequence was, thus, to create a new ECMO center within our existing mixed ICU, and this helped us to avoid various administrative barriers related to the hospital.

Guidelines recommend that ECMO centers must meet specific criteria, including creating a team consisting of different specialists, so that various specialists are directly involved in the patient's daily treatment. Mobile ECMO teams in these types of centers are also multidisciplinary [19-21].

In our center, the ECMO team is comprised only of the intensivists from the existing mixed ICU. No other specialists, such as cardiac and cardiothoracic surgeons or cardiologists, are engaged in the routine daily activity of the ECMO center. Moreover, perfusionists are not involved in the ECMO procedures at all. Therefore, we define our method of ECMO center creation as an 'ICU-based' approach.

In an ICU-based approach, it is assumed that an ECMO patient is usually affected by multi-organ failure, and this is comparable to other complex patients in intensive care. Consequently, and unlike in multidisciplinary-built ECMO centers, intensivists are responsible for most ECMO-related procedures, such as catheterizing vessels, priming the circuit, daily TTE/TEE examination, and routine checking of oxygenators. An ICU-based approach relieves the responsibility from external specialists, who can thus dedicate themselves to their main tasks, i.e., surgery. This model could also be used when the conditions for establishing a of multidisciplinary center are insufficient, e.g., lack of perfusionists, shortage of cardiothoracic surgeons, or intra-hospital administrative barriers. Table 4 highlights the main features of the multidisciplinary approach and our ICU-based approach to ECMO center organization.

Table 4. Methods for organizing the ECMO center.

\begin{tabular}{|c|c|c|}
\hline & Multidisciplinary Approach & ICU-Based Approach \\
\hline Staff & Specialists from different hospital wards & Entirely ICU personnel \\
\hline Leadership & Possibly a director from outside the ICU & Leader from the staff of the ICU \\
\hline Who treat & Purpose-built multidisciplinary team & Intensivists, consultants only upon request \\
\hline Who for & Only ECMO patients & One of the routine modalities in the ICU \\
\hline Administrative structure & A separate structure in the hospital & An integral part of an existing ICU \\
\hline Premises & Possibly external to the ICU & Only premises of the ICU \\
\hline
\end{tabular}

A few studies have shown an improvement in patient outcomes after the creation of multidisciplinary ECMO team in comparison with the pre-ECMO team period. Na et al. indicated that creating a multidisciplinary team mostly contributed to favorable outcomes [22]. Hong et al. stressed the importance of their multidisciplinary approach for an improvement in outcomes of patients being treated with VA-ECMO [23]. However, several key factors in both of these studies were lacking in the pre-ECMO periods, including protocols of ECMO process, a referral system, and staff training.

Interestingly, Komindr et al. did not report any improvement in patients' survival rate, ECMO duration, and hospital length of stay after the institution of a formal multidis- 
ciplinary ECMO team [24]. They argued that this was caused by the relatively high level of competencies in extracorporeal procedures among the staff already found during the pre-ECMO team period.

Whether the specific type of ECMO team (multidisciplinary or homogenous) matters is, thus, still not clear. The nature of the ECMO team is not necessarily the primary issue in terms of patient outcomes. Other aspects may affect the quality of care, including organized treatment, trained staff, designated leader of the team, clear protocols for ECMO inclusion, and the referral system.

Consequently, which type of ECMO center (multidisciplinary or ICU-based) is better for patients is still an unknown. Currently, a multidisciplinary approach is regarded as the 'gold' standard for optimum results. Notwithstanding, when extracorporeal therapy is not available at all in a region, a team consisting only of intensivists can both sufficiently perform ECMO procedures and form an efficient ECMO center within a mixed ICU. Based on our study, we also assume that intensivists can simply incorporate extracorporeal therapy into other routine modalities within the intensive care environment. A similar situation occurred in the 1990s, regarding the implementation of continuous renal replacement therapy (CRRT) in intensive care [25].

\section{Limitations}

Our approach has several limitations. First, only involving the staff from the ICU in the center's activity may lead to overburdening of the intensivists. Second, we do not perform extracorporeal therapy on potential donors. This limitation mainly stems from the legal situation regarding this type of therapy conducted among donors in Poland. Third, our center only performs extracorporeal therapy for the adult population. The ICU for neonates and children exists in the hospital as an entirely separate structure with dedicated staff. Fourth, for external patients only VV-ECMO is applied, by our mobile ECMO team. Except for intra-hospital eCPR, the application of VA-ECMO in the district ICU seems to be a more demanding procedure, regarding the inaccessibility of crucial facilities in district hospitals, such as a cath lab, vascular surgeon, or cardiac surgeon. Fifth, the small number of patients precludes performing both a detailed statistical analysis and an examination of the influence of various factors on the outcomes.

\section{Conclusions}

Organizing an operative ECMO center entirely on the basis of an existing mixed ICU is feasible. Although a multidisciplinary approach is still the 'gold' standard, an ICU-based approach can be implemented, leading to a practically functional regional ECMO center. Due to the small number of patients, further studies are needed to show that the ICU-based approach is no less effective than the multidisciplinary approach.

Author Contributions: Conceptualization, R.G. and T.C.; Methodology, R.G.; Formal analysis, K.L. and R.G.; Investigation, T.C., R.G., M.P. (Maciej Piwoda), M.M. (Maciej Marszalski) and M.M. (Maciej Molsa); Resources, T.C., R.G., M.P. (Maciej Piwoda), M.M. (Maciej Marszalski), M.G., J.P., M.M. (Magdalena Maj), M.M. (Maciej Molsa) and M.P. (Marek Pietka); Data curation, R.G. and T.C.; Writing — original draft preparation, R.G.; Writing—review and editing, R.G. and T.C. All authors have read and agreed to the published version of the manuscript.

Funding: This research received no external funding.

Institutional Review Board Statement: The study was conducted according to the guidelines of the Declaration of Helsinki and approved by the Regional Ethics Committee (date of approval: 6 June 2021, decision number: 335/2021).

Informed Consent Statement: Patient consent was waived due to the retrospective design of the study.

Data Availability Statement: The data presented in this study are available on request from the corresponding author. 
Conflicts of Interest: The authors declare no conflict of interest.

\section{References}

1. Ratnani, I.; Tuazon, D.; Zainab, A.; Uddin, F. The role and impact of extracorporeal membrane oxygenation in critical care. Methodist Debakey Cardiovasc. J. 2018, 14, 110-119. [CrossRef] [PubMed]

2. Extracorporeal Life Support Organization: ECLS Registry Report. Available online: https://www.elso.org/Registry/Statistics. aspx (accessed on 21 September 2021).

3. Barbaro, R.P.; Odetola, F.O.; Kidwell, K.M.; Paden, M.L.; Bartlett, R.H.; Davis, M.M.; Annich, G.M. Association of hospital-level volume of extracorporeal membrane oxygenation cases and mortality. Analysis of the extracorporeal life support organization registry. Am. J. Respir. Crit. Care 2015, 191, 894-901. [CrossRef] [PubMed]

4. Becher, P.M.; Goßling, A.; Schrage, B.; Twerenbold, R.; Fluschnik, N.; Seiffert, M.; Bernhardt, A.M.; Reichenspurner, H.; Blankenberg, S.; Westermann, D. Procedural volume and outcomes in patients undergoing VA-ECMO support. Crit. Care 2020, 24, 291. [CrossRef] [PubMed]

5. Friedrichson, B.; Mutlak, H.; Zacharowski, K.; Piekarski, F. Insight into ECMO, mortality and ARDS: A nationwide analysis of 45,647 ECMO runs. Crit. Care 2021, 25, 38. [CrossRef]

6. Barbaro, R.P.; MacLaren, G.; Boonstra, P.S.; Iwashyna, T.J.; Slutsky, A.S.; Fan, E.; Bartlett, R.H.; Tonna, J.E.; Hyslop, R.; Fanning, J.J.; et al. Extracorporeal membrane oxygenation support in COVID-19: An international cohort study of the extracorporeal life support organization registry. Lancet 2020, 396, 1071-1078. [CrossRef]

7. ELSO. ELSO Guidelines for ECMO Centers. Available online: https://www.elso.org/Resources/Guidelines.aspx (accessed on 21 September 2021).

8. Abrams, D.; Garan, A.R.; Abdelbary, A.; Bacchetta, M.; Bartlett, R.H.; Beck, J.; Belohlavek, J.; Chen, Y.S.; Fan, E.; Ferguson, N.D.; et al. Position paper for the organization of ECMO programs for cardiac failure in adults. Intensive Care Med. 2018, 44, 717-729. [CrossRef]

9. Combes, A.; Brodie, D.; Bartlett, R.; Brochard, L.; Brower, R.; Conrad, S.; De Backer, D.; Fan, E.; Ferguson, N.; Fortenberry, J.; et al. Position paper for the organization of extracorporeal membrane oxygenation programs for acute respiratory failure in adult patients. Am. J. Respir. Crit. Care 2014, 190, 488-496. [CrossRef]

10. DellaVolpe, J.; Barbaro, R.P.; Cannon, J.W.; Fan, E.; Greene, W.R.; Gunnerson, K.J.; Napolitano, L.M.; Ovil, A.; Pamplin, J.C.; Schmidt, M.; et al. Joint society of critical care medicine-extracorporeal life support organization task force position paper on the role of the intensivist in the initiation and management of extracorporeal membrane oxygenation. Crit. Care Med. 2020, 48, 838-846. [CrossRef]

11. Elkhwad, M.; More, K.S.; Anand, D.; Al-Maraghi, S.; Crowe, M.; Wong, D.; Metcalf, J.; Yadav, S.K.; Sigalet, D. Successful establishment of the first neonatal respiratory extracorporeal membrane oxygenation (ECMO) program in the middle east, in collaboration with pediatric services. Front. Pediatr. 2020, 8, 506. [CrossRef]

12. Cianchi, G.; Lazzeri, C.; Bonizzoli, M.; Batacchi, S.; Cozzolino, M.; Ciapetti, M.; Bernardo, P.; Franci, A.; Chiostri, M.; Peris, A. The 8-year experience of the florence referral ECMO center and retrieval team for acute respiratory failure. J. Cardiothorac. Vasc. Anesth. 2018, 32, 1142-1150. [CrossRef]

13. Combes, A.; Hajage, D.; Capellier, G.; Demoule, A.; Lavoué, S.; Guervilly, C.; Da Silva, D.; Zafrani, L.; Tirot, P.; Veber, B.; et al. Extracorporeal membrane oxygenation for severe acute respiratory distress syndrome. N. Engl. J. Med. 2018, 378, $1965-1975$. [CrossRef] [PubMed]

14. Shekar, K.; Badulak, J.; Peek, G.; Boeken, U.; Dalton, H.J.; Arora, L.; Zakhary, B.; Ramanathan, K.; Starr, J.; Akkanti, B.; et al. Extracorporeal life support organization coronavirus disease 2019 interim guidelines: A consensus document from an international group of interdisciplinary extracorporeal membrane oxygenation providers. Asaio J. 2020, 66, 707-721. [CrossRef] [PubMed]

15. Cotza, M.; Carboni, G.; Ballotta, A.; Kandil, H.; Isgrò, G.; Carlucci, C.; Varrica, A.; Garatti, A.; Giamberti, A.; Ranucci, M. Modern ECMO: Why an ECMO programme in a tertiary care hospital. Eur. Heart J. Suppl. 2016, 18 (Suppl. E), E79-E85. [CrossRef] [PubMed]

16. Shih, E.; DiMaio, J.M.; Squiers, J.J.; Banwait, J.K.; Meyer, D.M.; George, T.J.; Schwartz, G.S.; Baylor Scott \& White ECMO for COVID Group. Venovenous extracorporeal membrane oxygenation for patients with refractory Covid-19: Multicenter experience of referral hospitals in a large healthcare system. J. Thorac. Cardiovasc. Surg. 2020, 163, 1071-1079.e3. [CrossRef] [PubMed]

17. Patroniti, N.; Zangrillo, A.; Pappalardo, F.; Peris, A.; Cianchi, G.; Braschi, A.; Iotti, G.A.; Arcadipane, A.; Panarello, G.; Ranieri, V.M.; et al. The Italian ECMO network experience during the 2009 influenza A(H1N1) pandemic: Preparation for severe respiratory emergency outbreaks. Intensive Care Med. 2011, 37, 1447. [CrossRef]

18. Belohlávek, J.; Rohn, V.; Tosovsky, J.; Kunstyr, J.; Semrád, M.; Horák, J.; Lips, M.; Mlejnsky, F.; Vykydal, I.; Balík, M.; et al. A review of a newly established ECMO program in a university affiliated cardiac center. J. Cardiovasc. Surg. 2011, 52, 445-451.

19. Tipograf, Y.; Liou, P.; Oommen, R.; Agerstrand, C.; Abrams, D.; Brodie, D.; Bacchetta, M. A decade of interfacility extracorporeal membrane oxygenation transport. J. Thorac. Cardiovasc. Surg. 2019, 157, 1696-1706. [CrossRef]

20. Vaja, R.; Chauhan, I.; Joshi, V.; Salmasi, Y.; Porter, R.; Faulkner, G.; Harvey, C. Five-year experience with mobile adult extracorporeal membrane oxygenation in a tertiary referral center. J. Crit. Care 2015, 30, 1195-1198. [CrossRef]

21. Bonadonna, D.; Barac, Y.D.; Ranney, D.N.; Rackley, C.R.; Mumma, K.; Schroder, J.N.; Milano, C.A.; Daneshmand, M.A. Interhospital ECMO transport: Regional focus. Semin. Thorac. Cardiovasc. Surg. 2019, 31, 327-334. [CrossRef] 
22. Na, S.J.; Chung, C.R.; Choi, H.J.; Cho, Y.H.; Sung, K.; Yang, J.H.; Suh, G.Y.; Jeon, K. The effect of multidisciplinary extracorporeal membrane oxygenation team on clinical outcomes in patients with severe acute respiratory failure. Ann. Intensive Care $2018,8,31$. [CrossRef]

23. Hong, D.; Choi, K.H.; Cho, Y.H.; Cho, S.H.; Park, S.J.; Kim, D.; Park, T.K.; Lee, J.M.; Song, Y.B.; Choi, J.O.; et al. Multidisciplinary team approach in acute myocardial infarction patients undergoing veno-arterial extracorporeal membrane oxygenation. Ann. Intensive Care 2020, 10, 83. [CrossRef]

24. Komindr, A.; Abe, R.; Tateishi, Y.; Takahashi, Y.; Goto, J.; Wada, K.; Furukawa, Y.; Sugiura, A.; Imaeda, T.; Suga, N.; et al. Establishing extracorporeal membrane oxygenation team increased number of patients and improved data recording. J. Intensive Care 2019, 7, 11. [CrossRef]

25. Bellomo, R.; Cole, L.; Reeves, J.; Silvester, W. Who should manage CRRT in the ICU? The intensivist's viewpoint. Am. J. Kidney Dis. 1997, 30, S109-S111. [CrossRef] 